$\mathbb{T}$ periodica polytechnica

Civil Engineering

$53 / 2$ (2009) $61+74$

doi: 10.3311/pp.ci.2009-2.02

web: http://www.pp.bme.hu/ci

(c) Periodica Polytechnica 2009

RESEARCH ARTICLE

\section{FEM-based approach for the stability design of thin-walled members by using cFSM base functions}

\author{
Attila László Joó / Sándor Ádány
}

Received 2009-04-14, accepted 2009-05-09

\begin{abstract}
This paper presents a new design approach for the stability design of thin-walled members. The proposed approach is based on the buckling modes and critical forces/moments determined by a linear buckling analysis performed on a regular shell finite element model. A fully automatic buckling mode identification technique is applied, by using the modal base functions of the newly proposed constrained finite strip method, where the various buckling types are separated by clearly defined mechanical

criteria. The paper briefly summarizes the determination of modal base functions which then are used to approximate finite element displacement functions (i.e., buckling modes). The mode identification method provides the lowest critical values (forces or moments) to all the three characteristic buckling types: global, distortional and local, on the basis of which the buckling resistance can be calculated by using the design formulae of the direct strength method. The proposed new approach, which is potentially more general than any of the existing design approaches, is demonstrated on $Z$ columns and beams with simple loading and boundary conditions. Critical values as well as resistances are calculated for some selected cases, the results are compared to those of another design method. The comparisons prove the applicability of the proposed procedure. Further research is necessary to extend the proposal for more general and more complex practical cases.
\end{abstract}

\section{Keywords}

Design of thin-walled members $\cdot$ Buckling modes . Finite element method

\section{Attila László Joó}

Department of Structural Engineering, BME, Múegyetem rkp. 3. Budapest, H1521, Hungary

e-mail: ajoo@epito.bme.hu

\section{Sándor Ádány}

Department of Structural Mechanics, BME, Múegyetem rkp. 3. Budapest, H1521, Hungary

e-mail: sadany@epito.bme.hu

\section{Introduction}

An important tendency of the civil engineering industry during the last few decades is the application of more slender elements and structures. This trend is reflected e.g., by the wider and wider application of cold-formed steel members, supported by developments in the production technology as well as improvements in design methods, design standards and computational techniques. Cold-formed steel members are more and more used in Hungary, too, appearing in many innovative applications, such as light steel frames for small industrial buildings [1] and residential houses [2], light-weight trusses [3] or composite floor systems [4].

In the behaviour of thin-walled member buckling has crucial importance. It may take place in various forms, but usually three basic classes of buckling modes are distinguished: global, distortional, and local. Though widely accepted definitions for the classes do not exist, they are usually defined on the basis of inplane cross-sectional deformations. However, it is not the deformation pattern that makes the distinction important, but rather the post-buckling behaviour. Generally, global buckling has no post-buckling reserve, local buckling potentially has significant post-buckling reserve, and distortional buckling has moderate post-buckling reserve. Existence, or lack thereof, of postbuckling reserve greatly influences the member strength, thus it is important to be able to properly identify an arbitrary buckling mode.

Due to advances in computation, the calculation of buckling modes and critical forces is no longer a challenging task. For thin-walled members, most generally the problem is solved by the finite element method (FEM) using shell elements. However, FEM analysis typically leads to hundreds of buckling modes, most of them apparently interacted from modes of various classes. Given a lack of any quantitative method, the identification is typically done by visual inspection, which is a highly subjective process.

An alternative to FEM is the application of semi-analytical finite strip method (FSM), see e.g. [5-7]. By using FSM the critical values are to be calculated as a function of buckling length, and, based on this critical value vs. buckling length curve, also 


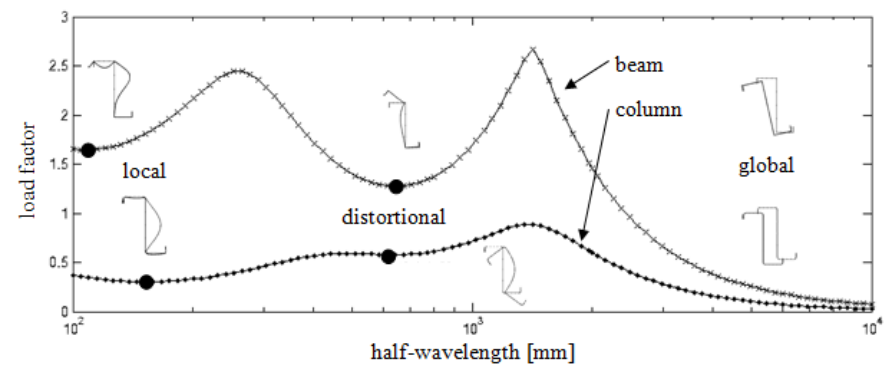

Fig. 1. Signature curves of a $\mathrm{Z}$ cross-section

known as the signature curve of the cross-section (see Fig. 1), the local, distortional or global critical loads can easily be determined for many practical cases: local and distortional values correspond to the two minimum points of the curve, while global value is most frequently the one calculated for the actual member length. A further practical advantage of FSM is that it is easily available, having been implemented into the open source CUFSM software [8]. Cases exist, however, when the shape of the signature curve makes the determination of local and/or distortional critical loads/stresses uncertain. Recently, a new version of FSM has been proposed which eliminates most of the uncertainties of the conventional FSM, see [9-13]. Mechanical criteria are defined and implemented into the semi-analytical FSM, through which the general displacement field of the FSM can be limited and the member can be enforced to deform according to pre-defined mechanical criteria, thus, to determine any "pure" buckling mode (e.g., global, distortional or local) or arbitrary combination of interacted modes. Since the embedded mechanical criteria mean constraints for the deformations, this special version of FSM is called constrained finite strip method (cFSM). The method is implemented into CUFSM software, too, providing a simple tool for the direct calculation of local, distortional and global critical values (or any combinations of them) for arbitrary open thin-walled cross-sections. Another useful feature of cFSM is that it can calculate the contribution of any individual buckling mode, or any mode class in an arbitrary FSM-calculated buckling (or deformation) mode. However, since cFSM is based on FSM, it naturally has all the limitations of FSM, e.g., the cross-section must be prismatic, only simple boundary conditions can correctly be handled, etc.

A possible approach to overcome the difficulties of both FEM and FSM/cFSM in the determination of pure global, distortional and local buckling modes and corresponding critical values is the combination of the FEM and cFSM by keeping the generality of FEM but utilizing the modal nature and mode separation capability of cFSM. Two approaches exist for the FEMcFSM combination. A possible approach is to implement constraints (similar to those used in cFSM) directly into FEM, which makes FEM capable to calculate any pure buckling mode (see for example: [14]). The other approach, followed here, uses the system of modal base functions of the cFSM to approximate displacements (i.e., buckling modes) calculated via un unconstrained FEM, e.g., by using shell finite elements. Since cFSM base functions are defined separately for the various mode classes, an approximate mode identification of general FEM buckling modes can be done. This mode identification method has been applied by the Authors, and has proved its applicability [15, 16], at least for certain cross-sections (namely: lipped channels), certain loading (namely: pure compression), and certain boundary conditions (namely: pinned-pinned end supports).

In this paper the mode identification method is applied for cases not studied earlier, namely: Z-section columns and beams, including illustrative example for a beam with discrete elastic restraints. Moreover, a new design approach is proposed on the basis of the mode identification method. The main steps of this design approach are as follows:

(i) Elastic buckling analysis of the considered thin-walled member by using the finite element method and shell finite elements. A fairly realistic model is possible to use, including practically any possible irregularities in the member (e.g., holes, certain cross-section changes), in the boundary conditions (e.g., intermediate and/or partial restraints), or in the loading.

(ii) Determination of the cFSM base functions.

(iii) Identification of the FEM calculated numerous buckling modes (e.g., first 50 modes). The identification is completed by approximating the FEM displacement functions as the linear combination of cFSM base functions. Since these cFSM base functions are given separately for the characteristic buckling types (e.g., global, local, distortional), their linear combination immediately yields to mode contribution percentages, i.e., it is possible to calculate the contribution of global, local or distortional class in an actual buckling mode.

(iv) Based on the critical values and the mode class contributions, the nominal "pure" modes can be selected, and the design resistance can be calculated by using a design standard.

In the subsequent Sections of the paper these main steps are briefly presented and applied for Z-section columns and beams. Based on the results conclusions are drawn and the potential further development of the proposed design approach is summarized.

\section{Modal base functions}

\subsection{General}

Determination of the modal base functions is in accordance with [5-7], and can be completed by the following steps: (i) a displacement field identical to the one used in finite strip method (FSM) is assumed, (ii) the characteristic buckling mode classes are defined by simple mechanical criteria, (iii) the mechanical 
criteria are implemented to separate the general FSM displacement field, and (iv) the resulting base system is orthogonalized and normalized.

For the analyzed member we assume that: (i) the member is a column, (ii) the column is prismatic, (iii) it is supported by two hinges at its ends, (iv) it is loaded by a compressive force (uniformly distributed along the cross-section), (v) its material is linearly elastic, and (vi) it is free from imperfections (residual stresses, initial deformations, material inhomogenities, etc.). It is important to point out that even though the determination of cFSM base functions is done on an axially loaded column, the base functions then can be used for beam or beam-column problems, too.

\subsection{FSM assumptions}

Since the cFSM is the specialization of FSM, the basic assumptions of FSM are reflected in the cFSM base functions/vectors. The implied assumptions can be summarized as follows: (i) the member is modeled by 2D surface elements, (ii) in-plane (membrane) and out-of-plane (plate bending) deformations are allowed, (iii) for the in-plane behavior a classical 2D stress state membrane is considered, (iv) for the out-of-plane behavior a classical Kirchhoff plate is considered, (v) the displacement functions are expressed as the product of nodal displacements (collected in displacement vectors) and shape functions, (vi) the transverse displacement functions are approximated by cubic polynomials, (v) and finally, the longitudinal displacement distribution is assumed in trigonometric form, as follows:

$$
\delta_{\text {transv }}=\delta_{\mathrm{CS}, \mathrm{t}} \sin \frac{m \pi y}{L} \text { and } \delta_{\text {longit }}=\delta_{\mathrm{CS}, \mathrm{w}} \cos \frac{m \pi y}{L}
$$

where $L$ is the member length, $m$ is the number of half-sinewaves considered, while $\delta_{\text {transv }}$ and $\delta_{\text {longit }}$ denotes (symbolically) the transverse and longitudinal displacement function, respectively, $\delta_{\mathrm{CS}, \mathrm{t}}$ and $\delta_{\mathrm{CS} \text {, w }}$ stand for cross-section transverse and warping displacements, respectively.

It is to observe that (i) the above functions correspond to pinned-pinned and free to warp boundary conditions, (ii) transverse displacements have maximum values in the middle of the beam, and (iii) longitudinal displacement has its maximum value at the beam ends.

\subsection{Definition of buckling modes}

In the literature and design standards for thin-walled members, it is common to distinguish three characteristic classes of buckling: global, distortional and local. Though there seems to be an agreement on the existence of these mode classes, there is no widely agreed upon definitions for them. It is also to mention that there evidently exist modes which neither can be categorized into any of the above three classes nor can be regarded as interaction of these three classes: these modes will be referred simply as other modes (including membrane shear and transverse extension).
Tab. 1. Mode class definition in $\mathrm{cFSM}$

\begin{tabular}{lcccc}
\hline & G mode class & D mode class & L mode class & O mode class \\
\hline Criterion 1 & Yes & Yes & Yes & No \\
\hline Criterion 2 & Yes & Yes & No & - \\
\hline Criterion 3 & Yes & No & - & - \\
\hline
\end{tabular}

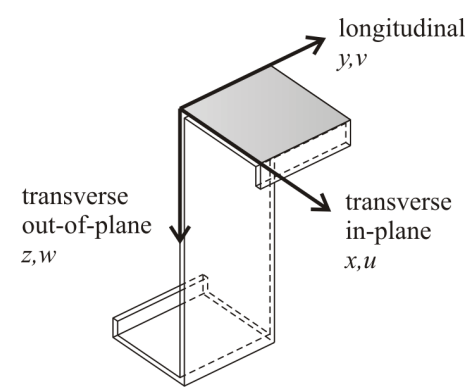

Fig. 2. Coordinates, displacements

Given the lack of commonly adopted mode definition, the one proposed in [11,12] is applied here. Note this definition can be regarded as equivalent to the one which is implicitly used in generalized beam theory (GBT), see e.g. [17, 18]. The separation between global (G), distortional (D), local (L) and other (O) deformation modes can be completed by the application of three mechanical criteria. Table 1 shows the criteria that must be satisfied by the different mode classes. By using the local coordinate system shown in Fig. 2, the criteria are as follows:

(i) Criterion 1: (a) $\gamma_{x y}=0$, i.e. there are no in-plane shear strains, (b) $\varepsilon_{x}=0$, i.e. there is no transverse extensions, and (c) $v$ is linear in $\mathrm{x}$ within a flat part.

(ii) Criterion 2: (a) $v \neq 0$, i.e. the warping displacement is not constantly equal to zero along the whole cross-section, and (b) the cross-section is in transverse equilibrium.

(iii) Criterion 3: $\kappa_{x x}=0$, i.e. there is no transverse flexure.

\section{4 cFSM base functions}

As it shown e.g. in [11], the above mechanical criteria can systematically be applied within the semi-analytical finite strip method (FSM). The application requires lengthy mathematical derivations, not discussed here, which finally lead to a base system where the various deformation classes are separated from each other. Since this separation can also be interpreted as application of appropriate constraints that enforce deformations according to the given criteria, this version of FSM is termed as constrained FSM, or cFSM.

The above mechanical criteria for mode definition unambiguously define subspaces of the original FSM displacement field, (also referred as G, D, L and O spaces), however, these spaces are typically multi-dimensional, therefore various systems of base functions are possible. According to the most basic idea of FSM, displacement functions are represented by vectors of nodal displacements, therefore, base functions are represented 
by nodal displacement vectors, too, hence simply referred as base vectors.

The application of the mechanical criteria of Table 1 leads to a constraint matrix, $\mathbf{R}$, for each deformation mode class, namely, $\mathbf{R}_{\mathrm{G}}, \mathbf{R}_{\mathrm{D}}, \mathbf{R}_{\mathrm{L}}$ and $\mathbf{R}_{\mathrm{O}}$. These $\mathbf{R}_{\mathrm{M}}$ matrices can also be interpreted as transformation matrices that make the transformation from the original FSM nodal system to the modal system of cFSM. Each $\mathbf{R}_{\mathrm{M}}$ defines a subspace consistent with a given deformation mode class and the columns of $\mathbf{R}_{\mathrm{M}}$ are the linearly independent base vectors for that subspace. Since displacement vectors always represent displacement functions, the columns of $\mathbf{R}_{\mathrm{M}}$ define linearly independent base functions for the sub-space. The G, D, L and O subspaces are nearly always multi-dimensional, thus, an infinite number of base vectors (functions) is possible to define within the subspace. However, if mode contributions are to be calculated, it is strongly advantageous to use orthonormal base vectors (functions). Thus, both orthogonalization and normalization should be performed.

According to cFSM, orthogonalization is completed by solving the constrained eigen-value problem for the member, separately for all four sub-spaces:

$$
\mathbf{R}_{\mathrm{M}}^{T} \mathbf{K}_{\mathrm{e}} \mathbf{R}_{\mathrm{M}} \boldsymbol{\Phi}_{\mathrm{M}}=\Lambda_{\mathrm{M}} \mathbf{R}_{\mathrm{M}}^{T} \mathbf{K}_{\mathrm{g}} \mathbf{R}_{\mathrm{M}}
$$

or

$$
\mathbf{K}_{\mathrm{eM}} \boldsymbol{\Phi}_{\mathrm{M}}=\Lambda_{\mathrm{M}} \mathbf{K}_{\mathrm{gM}}
$$

where subscript M may be G, D, L or O, $\mathbf{K}_{\mathrm{e}}$ and $\mathbf{K}_{\mathrm{g}}$ are the elastic and geometric stiffness matrices used in FSM for the given member, $\boldsymbol{\Phi}_{M}$ is the matrix of eigen-vectors, $\boldsymbol{\Lambda}_{M}$ is the diagonal matrix of the eigen-values, and $\mathbf{K}_{\mathrm{eM}}$ and $\mathbf{K}_{\mathrm{gM}}$ matrices may be interpreted as the constrained elastic and geometric stiffness matrices, respectively. As a result of Eq. (3), the system of the original base vectors, the columns of $\mathbf{R}_{\mathrm{M}}$, are transformed into a system of orthogonal base vectors, the columns of $\boldsymbol{\Phi}_{\mathrm{M}}$.

As it is proven in [11] the number of cross-section deformed configurations is dependent on the number of strips or nodes, and for an open unbranched cross-section it can be given as follows: $\mathrm{G}$ modes: $n_{\mathrm{G}, \mathrm{CS}}=4$, D modes: $n_{\mathrm{D}, \mathrm{CS}}=n m-4, \mathrm{~L}$ modes: $n_{\mathrm{L}, \mathrm{CS}}=n m+2 \times n s+2$, O modes: $n_{\mathrm{O}, \mathrm{CS}}=2 \times$ $n m+2 \times n s-2$, where $n m$ is the number of main cross-section nodes (at the corners) and $n s$ is the number of sub-nodes (or intermediate nodes) located between main nodes.

Eq. (1) suggests the total number of base functions depends also on the longitudinal shape function, namely: the $m$ number of half-sinewaves considered. Theoretically, $m$ can arbitrarily be selected. Practically, the number is limited to a certain $m_{\max }$ which provides reasonable accuracy for the approximation, when considering the wave-lengths $m=1 \cdots m_{\max }$. This means that the total number of base functions for a given mode is: $n_{\mathrm{M}}=n_{\mathrm{M}, \mathrm{CS}} \times m_{\max }$, where subscript $\mathrm{M}$ stands for $\mathrm{G}$, $\mathrm{D}, \mathrm{L}$ or $\mathrm{O}$. (Considering that the displacement functions that are to be approximated come from a finite element analysis, they are strongly dependent on the applied discretization. This suggests that the shortest considered sine half-wavelength should be comparable to the size of the applied finite elements, see also in Sec. 5])

\subsection{Normalization}

A fundamental feature of eigen-functions (eigen-vectors) is that they can be scaled arbitrarily. Since our aim here is to use the above-defined base functions to approximate displacement functions and then to determine contribution of the various mode classes, appropriate normalization (i.e., scaling) is indispensable. Various normalizations are possible. Here the simplest is used, the displacement vectors are normalized in a vector sense (taking advantage that displacement functions are expressed as the product of displacement vectors and shape functions). Vector normalization yields to reasonable results if a regular crosssection discretization is used. (Other normalizations are briefly discussed for cFSM in [13]).

For vector normalization, the $\boldsymbol{\Phi}_{\mathrm{M}, i}$ column vectors of the $\boldsymbol{\Phi}_{\mathrm{M}}$ matrices are normalized in a vector sense so that each (orthogonal and normalized)

$$
\boldsymbol{\varphi}_{i}=\frac{\boldsymbol{\Phi}_{\mathrm{M}, i}}{\left\|\boldsymbol{\Phi}_{\mathrm{M}, i}\right\|}
$$

vector satisfies that

$$
\boldsymbol{\varphi}_{i}^{T} \boldsymbol{\varphi}_{i}=1
$$

Though this normalization is not perfect (e.g. it is dependent on the applied discretization, the effect of rotations is underrepresented, etc.) similar normalization is used in GBT [18].

\section{Approximation of FEM solution by cFSM modes}

\subsection{Determination of coefficients in the linear combination}

Once the $\varphi$ cFSM base functions are known, it is possible to approximate any $\delta_{\mathrm{FE}}$ FEM displacement function as the linear combination of the base functions. The solution to get the cFSM approximation of a FEM displacement function is presented in [14, 15], and can be summarized as follows.

In order to have the best approximation, the error should be minimized as follows:

$$
\delta_{\mathrm{err}}=\delta_{\mathrm{FE}}-\sum c \varphi=\min !
$$

where $\sum c \varphi$ marks (symbolically) the linear combination. Following the logic used in normalization of the base functions (as discussed above), the minimization will be completed on the error vector (instead of error function), by minimizing the vector norm as follows:

$$
\mathbf{d}_{\mathrm{err}}^{T} \mathbf{d}_{\mathrm{err}}=\min !
$$

Considering that the error can be expressed as the difference of finite element displacement vector $\left(\mathbf{d}_{\mathrm{FE}}\right)$ and its approximation, i.e., the linear combination of the cFSM base vectors, Eq. (7) can be written as:

$$
\left(\mathbf{d}_{\mathrm{FE}}-\boldsymbol{\Phi} \mathbf{c}\right)^{T}\left(\mathbf{d}_{\mathrm{FE}}-\boldsymbol{\Phi} \mathbf{c}\right)=\min !
$$


where $\boldsymbol{\Phi}$ is the matrix with the FSM base vectors and $\mathbf{c}$ is the vector of unknown combination factors. With some further rearrangement:

$$
\mathbf{d}_{\mathrm{FE}}^{T} \mathbf{d}_{\mathrm{FE}}-(\boldsymbol{\Phi} \mathbf{c})^{T} \mathbf{d}_{\mathrm{FE}}-\mathbf{d}_{\mathrm{FE}}^{T} \boldsymbol{\Phi} \mathbf{c}+(\boldsymbol{\Phi} \mathbf{c})^{T} \boldsymbol{\Phi} \mathbf{c}=\min !
$$

Thus, the function to be minimized can be expressed as follows:

$$
f(\mathbf{c})=\mathbf{d}_{\mathrm{FE}}^{T} \mathbf{d}_{\mathrm{FE}}-2 \boldsymbol{\Phi}^{T} \mathbf{d}_{\mathrm{FE}} \mathbf{c}+\mathbf{c}^{T} \boldsymbol{\Phi}^{T} \boldsymbol{\Phi} \mathbf{c}=\min !
$$

Minimization finally leads to a linear system of equations to be solved for $\mathbf{c}$ :

$$
\begin{gathered}
\frac{\partial f(\mathbf{c})}{\partial \mathbf{c}}=0 \\
\boldsymbol{\Phi}^{T} \boldsymbol{\Phi} \mathbf{c}=\boldsymbol{\Phi}^{T} \mathbf{d}_{\mathrm{FE}} .
\end{gathered}
$$

\subsection{Mode participation calculation}

After calculating the combination factors, $p_{i}$ participation of an individual buckling mode (or base function) can be calculated:

$$
p_{i}=\left|c_{i}\right| / \sum_{\text {all }}\left|c_{i}\right| .
$$

Taking advantage that within the base functions the various buckling classes (i.e. global, distortional or local) are separated, the $p_{\mathrm{M}}$ participation of a class can be calculated as the sum of the individual participation of the base vectors from the given mode class:

$$
p_{\mathrm{M}}=\sum_{\mathrm{M}}\left|c_{i}\right| / \sum_{\text {all }}\left|c_{i}\right|
$$

where $c_{i}$ is an element of $\mathbf{c}$ vector, while $\mathrm{M}$ denotes that summation should be over all elements of a given mode class.

\subsection{Error of approximation}

Once the FEM displacement function is expressed by the cFSM base functions, the error of approximation can conveniently be measured as the norm of the error vector relative to the norm of the displacement vector:

$$
e r r=\sqrt{\mathbf{d}_{\mathrm{err}}^{T} \mathbf{d}_{\mathrm{err}}} / \sqrt{\mathbf{d}_{\mathrm{FE}}^{T} \mathbf{d}_{\mathrm{FE}}} .
$$

\subsection{Determination of critical forces/moments for the mode} classes

By solving Eq. (12), any of the FEM calculated multiple buckling modes can be approximated by the cFSM base functions, thus, contribution of G, D, L and $\mathrm{O}$ classes can be calculated by using Eq. (14). The practical experience is that clearly pure modes rarely exist, which means that $100 \%$ contribution of any mode class practically never happens. This is especially true for D mode class: even in seemingly pure distortional buckling cases the D contribution rarely more than $80 \%$. Another practical experience is that the contribution of $\mathrm{O}$ modes frequently non-negligible, due to the applied mode class definition and orthonormalization.

Since we need to select "pure" buckling modes, but in a strict mathematical sense "pure" modes do not exist, it is proposed to define nominal pure modes in a more engineering way, by adopting any mode as "pure" if the contribution from a given mode class is at least $70-80 \%$. Thus, the critical value for a given mode class will be the smallest one that belong to a buckling mode in which the contribution of the given mode class is at least $70-80 \%$. (Note, the presented numerical studies prove the applicability of this definition of pure critical forces/moments, see Section 5 Nevertheless further studies are necessary on this question.)

\section{Design resistance by the Direct Strength Method 4.1 General}

Once the critical values (forces, moments, stresses) for global, local and distortional classes are known, the design resistance can be calculated. Different design codes give different approaches and formulae. The traditional way is the use of effective widths, as in Eurocode or AISI specification [19,20]. A more straightforward and relatively new proposal for the calculation of the design resistance of thin-walled (cold-formed) cross-section members is the so-called Direct Strength Method (DSM), see [21], which has been adopted by the North American Standard for cold-formed steel design [22, 23]. The idea of the DSM is to predict the resistance on the basis of elastic critical force or moment separately for the three characteristic buckling types, namely: global, distortional and local capacity. The member resistance will be simply the minimum of the three predicted capacities. The prediction of the global, distortional and local resistances is realized by simple equations, given separately for column and beam problems.

\subsection{Column formulae}

In case of columns, the $P_{\text {ne }}$ global Eq. 16, $P_{\text {nl }}$ local Eq. 17] and $P_{\text {nd }}$ distortional Eq. (18) capacities are given as follows:

$$
P_{\mathrm{ne}}=\left\{\begin{array}{lll}
\left(\frac{0.877}{\lambda_{\mathrm{c}}^{2}}\right) P_{\mathrm{y}} & \text { if } & \lambda_{\mathrm{c}}>1.5 \\
\left(0.658^{\lambda_{\mathrm{c}}^{2}}\right) P_{\mathrm{y}} & \text { if } & \lambda_{\mathrm{c}} \leq 1.5
\end{array}\right.
$$

$$
P_{\mathrm{nl}}=\left\{\begin{array}{lll}
\left(1-0.15\left(\frac{P_{\mathrm{crl}}}{P_{\mathrm{ne}}}\right)^{0.4}\right)\left(\frac{P_{\mathrm{crl}}}{P_{\mathrm{ne}}}\right)^{0.4} P_{\mathrm{ne}} & \text { if } \quad \lambda_{1}>0.776 \\
P_{\mathrm{ne}} & \text { if } \quad \lambda_{1} \leq 0.776
\end{array}\right.
$$

$P_{\text {nd }}=\left\{\begin{array}{lll}\left(1-0.25\left(\frac{P_{\text {crd }}}{P_{\mathrm{y}}}\right)^{0.6}\right)\left(\frac{P_{\text {crd }}}{P_{\mathrm{y}}}\right)^{0.6} P_{\mathrm{y}} & \text { if } & \lambda_{\mathrm{d}}>0.561 \\ P_{\mathrm{y}} & \text { if } \quad \lambda_{\mathrm{d}} \leq 0.561\end{array}\right.$

where the slendernesses Eq. 197 are:

$$
\lambda_{\mathrm{c}}=\sqrt{P_{\mathrm{y}} / P_{\mathrm{cre}}}, \quad \lambda_{1}=\sqrt{P_{\mathrm{ne}} / P_{\mathrm{crl}}} \quad \text { and } \quad \lambda_{\mathrm{d}}=\sqrt{P_{\mathrm{y}} / P_{\mathrm{crd}}}
$$

and $P_{\text {crl }}, P_{\text {crd }}$ and $P_{\text {cre }}$ are the critical axial forces for local, distortional and global buckling, respectively, and $P_{\mathrm{y}}$ is the squash 
load of the cross-section, which is equal to the product of the cross-sectional area and the yield strength.

Thus, the design capacity of the member for axial compressive force is the minimum of the predicted capacities:

$$
P_{\mathrm{n}}=\min \left(P_{\mathrm{ne}}, P_{\mathrm{nl}}, P_{\mathrm{nd}}\right) \text {. }
$$

\subsection{Beam formulae}

In case of beams the procedure is the same, with some differences in the formulae see Eqs. (21)- 23 .

$$
M_{\mathrm{ne}}=\left\{\begin{array}{lll}
M_{\text {cre }} & \text { if } \quad M_{\text {cre }}<0.56 M_{\mathrm{y}} \\
\frac{10}{9} M_{\mathrm{y}}\left(1-\frac{10 M_{\mathrm{y}}}{36 M_{\text {cre }}}\right) & \text { if } \quad 2.78 M_{\mathrm{y}} \geq M_{\text {cre }} \geq 0.56 M_{\mathrm{y}} \\
M_{\mathrm{y}} & \text { if } \quad M_{\text {cre }}>2.78 M_{\mathrm{y}}
\end{array}\right.
$$

$M_{\mathrm{nl}}=\left\{\begin{array}{lll}\left(1-0.15\left(\frac{M_{\mathrm{crl}}}{M_{\mathrm{ne}}}\right)^{0.4}\right)\left(\frac{M_{\mathrm{crl}}}{M_{\mathrm{ne}}}\right)^{0.4} M_{\mathrm{ne}} & \text { if } \quad \lambda_{1}>0.776 \\ P_{\mathrm{ne}} & \text { if } \quad \lambda_{1} \leq 0.776\end{array}\right.$

$M_{\mathrm{nd}}=\left\{\begin{array}{lll}\left(1-0.22\left(\frac{M_{\text {crd }}}{M_{\mathrm{y}}}\right)^{0.5}\right)\left(\frac{M_{\text {crd }}}{M_{\mathrm{y}}}\right)^{0.5} M_{\mathrm{y}} & \text { if } & \lambda_{\mathrm{d}}>0.673 \\ M_{\mathrm{y}} & \text { if } & \lambda_{\mathrm{d}} \leq 0.673\end{array}\right.$

where the slendernesses Eq. 24) are:

$$
\lambda_{\mathrm{l}}=\sqrt{M_{\mathrm{ne}} / M_{\mathrm{crl}}} \text { and } \lambda_{\mathrm{d}}=\sqrt{M_{\mathrm{y}} / M_{\mathrm{crd}}}
$$

and $M_{\mathrm{crl}}, M_{\mathrm{crd}}$ and $M_{\mathrm{cre}}$ are the critical bending moments for local, distortional and global buckling, respectively, and $M_{\mathrm{y}}$ is the yield moment of the cross-section, which is equal to the product of the elastic section modulus and the yield strength.

Thus, the design capacity of the member for bending moment is the minimum of the predicted capacities:

$$
M_{\mathrm{n}}=\min \left(M_{\mathrm{ne}}, M_{\mathrm{nl}}, M_{\mathrm{nd}}\right) \text {. }
$$

It is to note that the above formulae are validated for the most frequently applied cold-formed cross-sections, including a wide range of $\mathrm{C}$ ad $\mathrm{Z}$ sections, Hat sections and Rack sections.

\section{Numerical studies}

\subsection{Background}

In this paper unsymmetrical Z-section members are studied. The examined cases are defined by utilizing the conclusions of earlier numerical studies on the proposed mode identification method. These earlier numerical studies have been carried out on C-shaped columns, presented in [14, 15], where the applicability of the proposed method is proven for compressed Csections. As it was demonstrated, the proposed identification method in its current form is applicable provided (i) the crosssection discretization is dense enough, (ii) the end supports are similar to or more rigid than a pinned supports, (iii) the minimal half-wave length of the cFSM base functions is not smaller than approximately twice the length of the shell finite elements, and (iv) the typical buckling length of the considered buckling mode is not smaller than the minimal half-wave length of the cFSM base functions considered.

\subsection{The analyzed Z-members}

The most important data of the analyzed models are summarized in Table 2 .

Three member lengths are considered: 800, 2000 and $3600 \mathrm{~mm}$. The cross-section dimensions are as follows: web height is $198 \mathrm{~mm}$, flange widths are 64 and $72 \mathrm{~mm}$, lip lengths are $19.8 \mathrm{~mm}$; thickness is $2 \mathrm{~mm}$, and the lips are perpendicular to flanges. (Note, the dimensions are for the mid-line, and sharp corners are employed.) Steel material is assumed with a Young's modulus of $210000 \mathrm{MPa}$ and Poisson's ratio of 0.3. (Note, these data follow those of specimens of an experimental program, carried out in the Structural Laboratory of the Budapest University of Technology and Economics, see [24].)

Two types of loading are considered: a concentric axial compressive force (applied as uniformly distributed pressure at the column ends) and a uniform bending moment (applied as linearly distributed loading at the beam ends).

As far as boundary conditions (BC) are concerned, in most of the analyzed cases they are defined to simulate pinned and free-to-warp end restraints. In case of shell finite elements such a condition can be realized in multiple ways (at least approximately). Here a rather theoretical solution is applied in order to closely imitate FSM conditions, i.e., to ensure coincidence with the mechanical assumptions underlying the FSM base functions. Namely, the following degrees of freedom (DOF) are restrained: transverse translational DOF of all the nodes at both column ends, and longitudinal translational DOF of the nodes at the middle of the member. (Note, this special restraining is still not perfectly identical to the one of FSM, since rotational DOF are not restrained, and since it is really only coincident with the boundary conditions for the case of one half-wavelength, i.e., $m=1$.)

Two types of other support conditions are also used. One is to model the case of a bolted or screwed connection to the member web, which ensures restraint against the out-of-plane and in-plane translations of the web nodes (referred as LW, standing for "local web"). The other one is a pinned-pinned end condition (basically similar to FSM), but an additional spring in lateral direction and torsional spring is introduced at middle of the member at the web-to-flange intersection of the model (one side only) to consider the restraining effect of an attached structural element. The three boundary conditions are demonstrated in Fig. 3

\section{$5.3 \mathrm{cFSM}$ base functions for the $\mathbf{Z}$ members}

Some of the orthogonal modes (i.e., cFSM base functions) are presented in Fig. 4 , where the characteristic deformed cross- 
Tab. 2. Summary of the analyzed Z-members

\begin{tabular}{|c|c|c|c|c|c|c|c|}
\hline Model & $\begin{array}{c}\text { Length } \\
\text { [mm] }\end{array}$ & $\mathrm{N}$ & $M$ & $\begin{array}{l}\text { Boundary } \\
\text { condition }\end{array}$ & $\begin{array}{l}\text { Cross-section } \\
\text { discretization } \\
\text { (flange/web/lip) }\end{array}$ & $\begin{array}{c}\text { \# of } \\
\text { element } \\
\text { long. }\end{array}$ & $\begin{array}{r}\text { Additional } \\
\text { spring at } \\
\text { half-span }\end{array}$ \\
\hline 1 & 800 & Yes & - & FSM & $3-5-1$ & 52 & - \\
\hline 2 & 800 & Yes & - & FSM & $3-5-1$ & 26 & - \\
\hline 3 & 800 & Yes & - & LW & $3-5-1$ & 26 & - \\
\hline 4 & 800 & - & Yes & FSM & $3-5-1$ & 26 & - \\
\hline 5 & 800 & - & Yes & FSM & $3-5-1$ & 52 & - \\
\hline 6 & 800 & - & Yes & FSM & $3-5-1$ & 78 & - \\
\hline 7 & 2000 & Yes & - & FSM & $3-5-1$ & 66 & - \\
\hline 8 & 2000 & Yes & - & FSM & $2-4-1$ & 108 & - \\
\hline 9 & 2000 & - & Yes & FSM & $3-5-1$ & 66 & - \\
\hline 10 & 2000 & - & Yes & FSM & $2-4-1$ & 108 & - \\
\hline 11 & 3600 & Yes & - & FSM & $3-5-1$ & 118 & - \\
\hline 12 & 3600 & - & Yes & FSM & $3-5-1$ & 118 & - \\
\hline 13 & 2000 & Yes & - & FSM & 2-4-1 & 66 & Yes \\
\hline
\end{tabular}

a)

a)

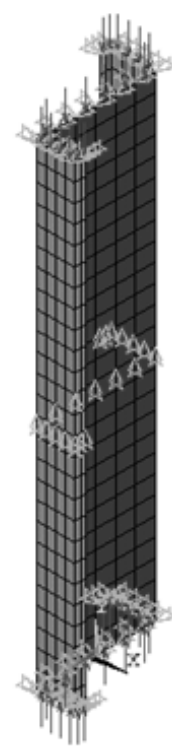

b)

b)

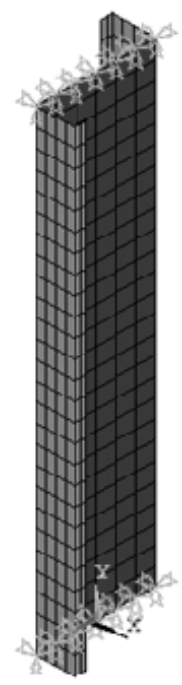

c)

c)

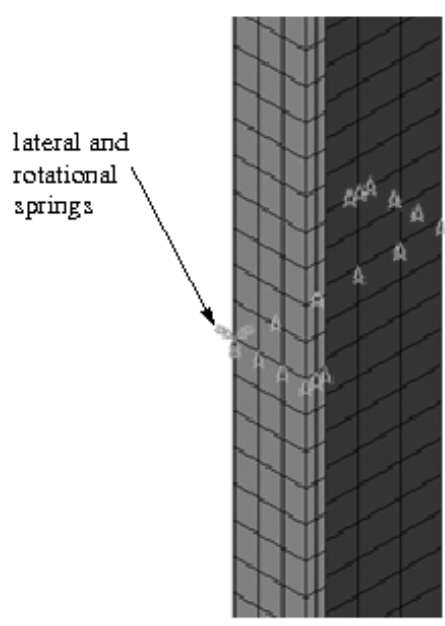

Fig. 3. Boundary conditions: a) FSM-like support, b) LW type support, c) FSM-like support with additional springs 
section shapes are shown. Note, these modes are in basic accordance with the ones used in GBT even though they are not identical to those.

As it is clear from the derivation of base functions, they are characterized by not only the deformed cross-section shape, but also by longitudinal wave-length. To illustrate this, Fig. 5 shows the base functions for one global buckling mode (namely: flexural mode) for various half-wave lengths (namely: $m=1$ to $5)$.

\subsection{Finite element buckling analysis}

For the linear buckling analysis the Ansys finite element software is applied [25]. The considered $\mathrm{Z}$ beams/columns are modeled by shell finite elements, namely: 4-node 24-DOF rectangular shell finite elements are used (called SHELL63 in Ansys terminology). The element numbers used in the models varied from 468-2124, depending on the member length and the mesh density.

To ensure accurate numerical results, flanges and web are necessary to discretize. Based on the conclusions of earlier studies, two mesh densities are considered: a finer mesh with 3 intermediate/sub nodes in each flange, 5 in the web and 1 in the lip (option 3-5-1) and a rare mesh with 2, 4 and 1 node, respectively (option 2-4-1). In the longitudinal direction various element lengths are tested. It is obvious that more elements in the longitudinal direction means more accurate numerical results. However, since buckling modes with very short buckling lengths (e.g., less than the widest plate element of the crosssection) rarely have practical relevance, it has been found that a relatively rare longitudinal discretization provides better computational efficiency while does not significantly reduces the numerical accuracy.

In the presented study usually the first 100 buckling modes and corresponding critical values have been determined. It is to note, however, that this number could significantly reduced, since the first 30-50 modes are found to be enough for practical purposes.

Table 3 presents the critical load factors for the first 20 buckling modes for model \#9, i.e., 2000-mm-long pinned-pinned beam subjected to uniform end-moment. (Note, the GDLO participation percentages are also given, as will be discussed in the subsequent Section.)

Similar tables have been generated for all the 13 analyzed models and for the first 100 buckling modes. It would not be possible neither reasonable to give all these tables in detail, therefore, only some characteristic and interesting buckling modes are presented. The buckled shapes are demonstrated in Figs. 68 for the selected models and buckling modes, for member lengths 800, 2000 and $3600 \mathrm{~mm}$, respectively. (Note, $3 \mathrm{D}$ views are shown on the top part of the figures, while crosssection deformations are shown on the bottom part.) The critical forces/moments (together with the GDLO participations) are summarized in Table 4
It might be interesting to point out some similarities and differences between the FSM and FEM solutions. For example, one might observe that the first FEM buckling mode for the 2000-mm-long beam is a practically pure distortional mode (Table 3, with three longitudinal waves as shown in Fig. 7 , d), thus, with a characteristic buckling length of approx. $670 \mathrm{~mm}$. The FSM-calculated signature curve for bending (see Fig. 1 ) clearly shows that the buckling mode is distortional for this specific length, as well as the good coincidence of the critical load factors is found. Similar observations apply for the second FEM buckling mode (lateral-torsional buckling, see also Fig. 7 e), or the $6^{\text {th }}$ FEM buckling mode (pure local buckling, see also Fig 7 f). At the same time there are several FEM buckling modes without obvious FSM counterparts, e.g., where various mode classes are combined (with different half-wave lengths).

\subsection{Mode participation calculation}

Mode participation calculation has been completed for all the cases and all the buckling modes, by solving the linear system of equations Eq. (11), then by using Eq. (13). Some of the results are shown in Tables 3 and 4. Based on the completed mode identification calculations the following remarks are to mention.

Generally, it may be observed that the calculated GDLO participation percentages are in accordance with the engineering judgment. In most of the buckling modes L contribution is significant, for shorter columns or beams almost all (if not all) the practically relevant buckling modes are local modes.

Calculation of the $\mathbf{c}$ linear combination factors, i.e., solving Eq. (12) has been completed by using MatLab [26]. In case of large number of degrees of freedom (such as in the case of longer member) the size of the problem means significant computational difficulty and computation time. (This problem could be solved by reducing the number of considered cFSM base function, e.g. not using all the cFSM base functions for the approximation of the FEM buckling mode, but by neglecting local base functions with small transverse waves and/or by neglecting some or all the $\mathrm{O}$ base functions. This reduction of modal base system, however, has not been applied in the presented study.)

In case of LW type supports, i.e., when web nodes are restrained only, the mode identification does not work properly. This finding is in full agreement with the conclusions of previous studies, LW support condition being "less rigid" than a hinge. The obvious reason the mode identification fails to work for these BC-s is the assumed longitudinal shape functions of the cFSM base functions, which clearly do not satisfy these types of BC-s, see Eq. (1). It is almost sure that the mode identification method can be generalized by using base functions worked out for different longitudinal shape functions.

It is worth to highlight two cases analysed for bending moment, as shown in Fig.77 c) and g). Both are for simple pinnedpinned beam, but part c) is without, while part g) is with extra springs at middle of the member. As one would expect: the applied (relatively light) springs hardly influence the local and 


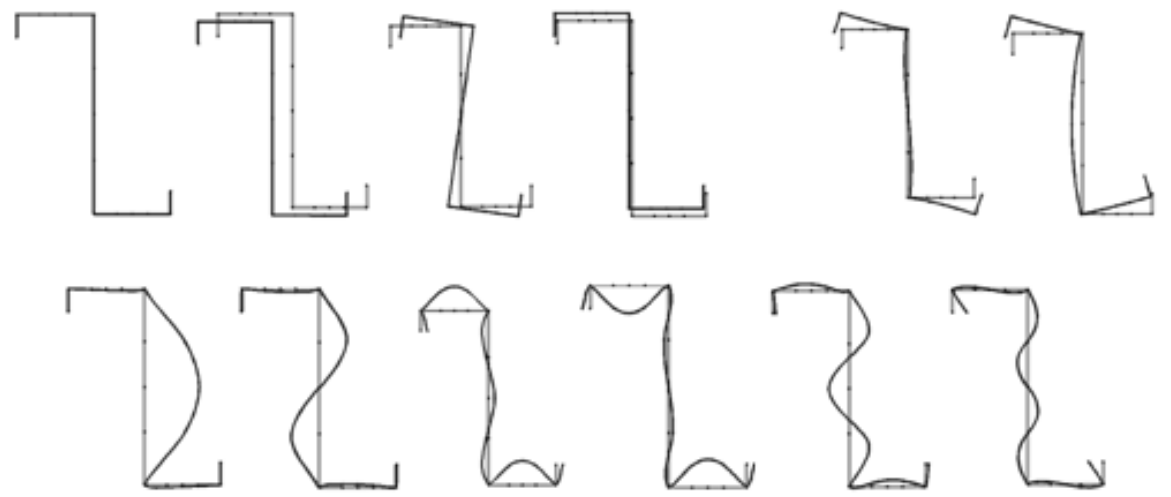

Fig. 4. Four global, two distortional and the first six local modes (displaced/deformed cross-sections)
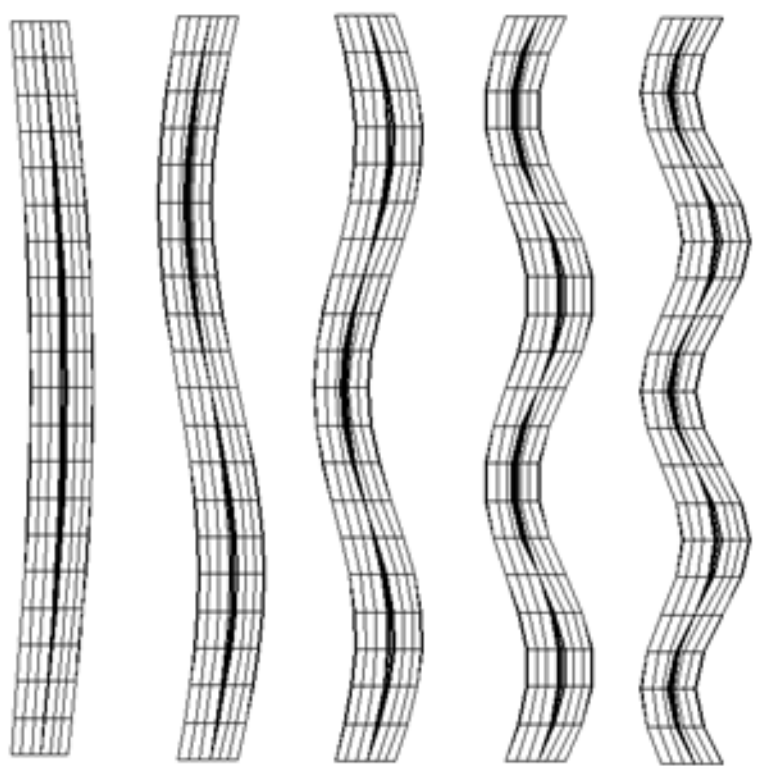

Fig. 5. A global (flexural) base function with various half-wave lengths

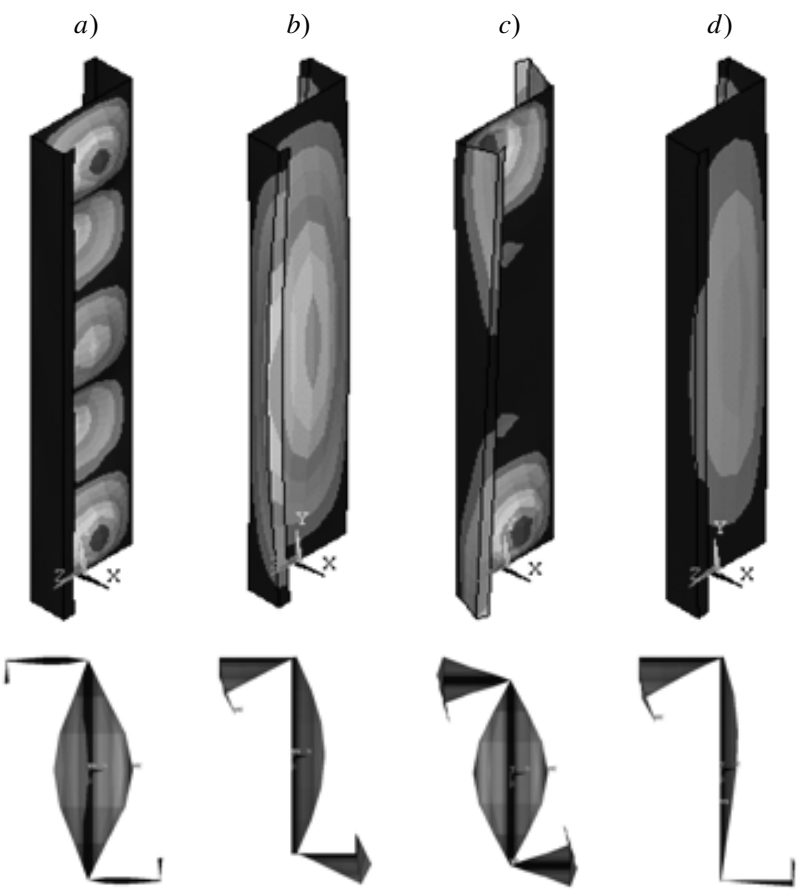

Fig. 6. Selected buckling modes of member length $800 \mathrm{~mm}$ : modes 1,11 of model \#1, mode 1 of model \#3 and \#6 

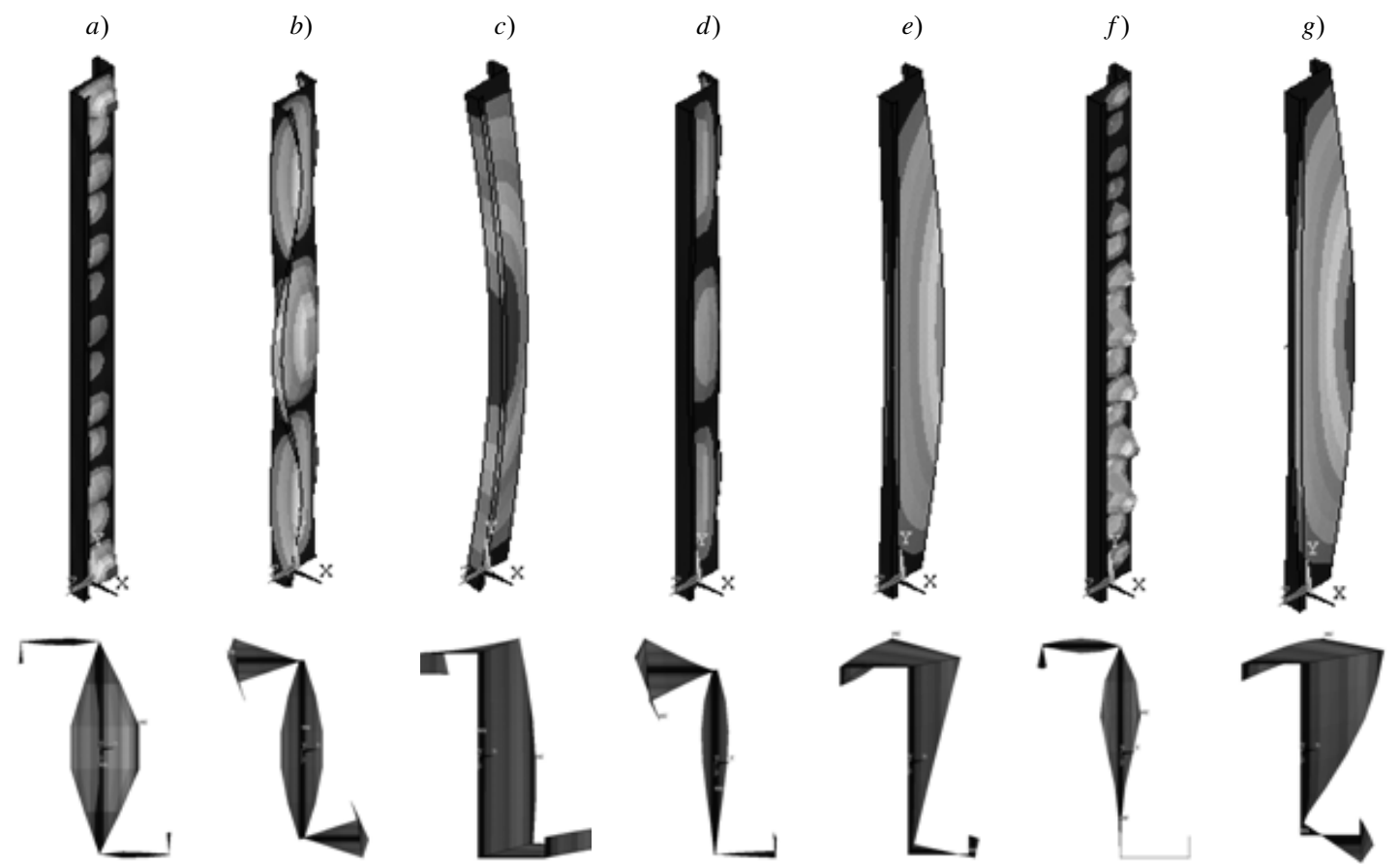

Fig. 7. Selected buckling modes of member length $2000 \mathrm{~mm}$ : modes 1, 26, 28 of model \#8, modes 1, 2, 6 of model \#9 and mode 40 of model \#13

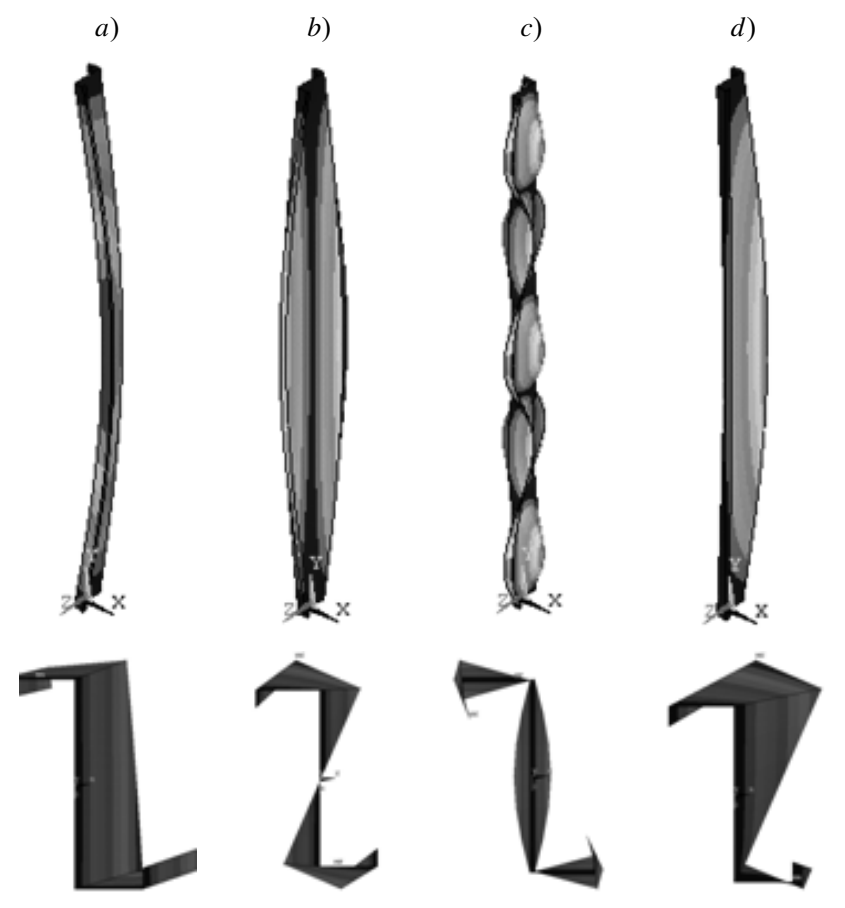

Fig. 8. Selected buckling modes of member length $3600 \mathrm{~mm}$ : modes 1, 39, 50 of model \#11 and mode 1 of model \#12 
Tab. 3. Critical values and GDLO participations for the first 20 buckling modes of model \#9 (i.e., 2000-mm-long simple supported beam)

\begin{tabular}{|c|c|c|c|c|c|c|}
\hline Mode \# & Critical load factor & Global [\%] & Distort. [\%] & Local [\%] & Other [\%] & Error [\%] \\
\hline 1 & 1.20 & 1.03 & 90.62 & 4.04 & 4.31 & 0.01 \\
\hline 2 & 1.38 & 84.56 & 5.16 & 1.02 & 9.26 & 0.00 \\
\hline 3 & 1.42 & 8.56 & 64.81 & 22.56 & 4.07 & 0.19 \\
\hline 4 & 1.43 & 4.82 & 56.30 & 35.46 & 3.42 & 0.32 \\
\hline 5 & 1.45 & 3.13 & 22.57 & 72.26 & 2.03 & 1.41 \\
\hline 6 & 1.46 & 0.69 & 6.82 & 91.28 & 1.21 & 0.38 \\
\hline 7 & 1.46 & 1.43 & 17.52 & 79.46 & 1.59 & 0.63 \\
\hline 8 & 1.47 & 0.94 & 15.00 & 82.57 & 1.49 & 0.48 \\
\hline 9 & 1.47 & 0.72 & 6.20 & 91.85 & 1.23 & 0.75 \\
\hline 10 & 1.49 & 1.22 & 15.63 & 81.64 & 1.52 & 0.97 \\
\hline 11 & 1.49 & 1.20 & 10.05 & 87.31 & 1.44 & 0.45 \\
\hline 12 & 1.51 & 0.54 & 9.19 & 88.99 & 1.28 & 1.12 \\
\hline 13 & 1.52 & 0.54 & 13.84 & 84.23 & 1.39 & 1.21 \\
\hline 14 & 1.53 & 0.95 & 19.11 & 78.37 & 1.57 & 0.39 \\
\hline 15 & 1.55 & 0.55 & 5.72 & 92.47 & 1.26 & 1.98 \\
\hline 16 & 1.57 & 0.42 & 8.60 & 89.69 & 1.29 & 2.65 \\
\hline 17 & 1.58 & 1.65 & 9.99 & 86.59 & 1.77 & 0.59 \\
\hline 18 & 1.60 & 0.12 & 6.32 & 92.18 & 1.39 & 2.62 \\
\hline 19 & 1.62 & 0.48 & 12.05 & 85.93 & 1.54 & 4.24 \\
\hline 20 & 1.63 & 0.85 & 74.09 & 18.40 & 6.65 & 0.06 \\
\hline
\end{tabular}

Tab. 4. Critical values and GDLO participations for the selected buckling modes

\begin{tabular}{|c|c|c|c|c|c|c|c|c|}
\hline Length [mm] & Model & Mode \# & Crit. factor & $\mathrm{G}[\%]$ & $\mathrm{D}[\%]$ & L [\%] & $\mathrm{O}[\%]$ & Error [\%] \\
\hline \multirow{4}{*}{800} & 1 & 1 & 0.29 & 0.36 & 6.68 & 91.86 & 1.11 & 0.01 \\
\hline & 1 & 11 & 0.61 & 6.08 & 79.79 & 10.65 & 3.49 & 0.00 \\
\hline & 3 & 1 & 0.18 & 0.38 & 3.92 & 27.88 & 67.82 & 36.04 \\
\hline & 6 & 1 & 1.29 & 1.04 & 93.43 & 3.28 & 2.24 & 0.01 \\
\hline \multirow{7}{*}{2000} & 8 & 1 & 0.29 & 0.55 & 5.38 & 92.83 & 1.23 & 0.02 \\
\hline & 8 & 26 & 0.58 & 6.08 & 74.91 & 14.13 & 4.88 & 0.01 \\
\hline & 8 & 28 & 0.59 & 74.71 & 13.31 & 1.79 & 10.19 & 0.01 \\
\hline & 9 & 1 & 1.20 & 1.03 & 90.62 & 4.04 & 4.31 & 0.01 \\
\hline & 9 & 2 & 1.38 & 84.56 & 5.16 & 1.02 & 9.26 & 0.00 \\
\hline & 9 & 6 & 1.47 & 0.69 & 6.82 & 91.28 & 1.21 & 0.38 \\
\hline & 13 & 40 & 0.92 & 76.26 & 12.43 & 2.60 & 8.71 & 0.01 \\
\hline \multirow{4}{*}{3600} & 11 & 1 & 0.19 & 53.14 & 0.11 & 0.14 & 46.61 & 0.54 \\
\hline & 11 & 39 & 0.50 & 64.56 & 1.05 & 0.61 & 33.77 & 0.08 \\
\hline & 11 & 50 & 0.59 & 5.49 & 75.33 & 12.31 & 6.87 & 0.03 \\
\hline & 12 & 1 & 0.41 & 90.74 & 0.43 & 0.09 & 8.74 & 0.00 \\
\hline
\end{tabular}


distortional buckling modes and critical forces, but significantly change the global buckling mode and significantly increase the critical force for the global mode (i.e., load factor is increased by the springs from 0.58 to 0.92 ).

Finally, it is interesting to highlight the non-negligible contribution of $\mathrm{O}$ modes in case of seemingly global buckling, which can be traced back to the fact that the applied mode class definition does not allow transverse extension of the plate elements except in $\mathrm{O}$ modes. (For more details see [11].)

\subsection{Design resistances}

Design resistances are calculated for both the column and beam problems, for all the three lengths, and in case of the 2000$\mathrm{mm}$-long beam also for the restrained middle section case where FSM buckling mode cannot be calculated due to the restrictions of the method. The resistance calculation is completed by using DSM formulae, as summarized in Sec. 4

First, the nominal pure critical values are to be determined, as summarized in Table 5. The nominal pure critical values are given as determined by FEM (via the mode identification method) and as determined by regular FSM analysis. It can be observed that there is no significant difference between the values provided by the two methods, which statement is in accordance with the conclusions of previous studies.

It is interesting to note that in case of the shortest $800-\mathrm{mm}$ long members global buckling occurs at very high critical values only. That is why neither FSM nor FEM analysis gave a definite critical value for global buckling. According to the logic of DSM, however, this does not cause any practical problem in the calculation, since very large critical force/moment means that the actual buckling mode is not a potential failure mode. Practically, a sufficiently large critical value can be assumed to ensure that the predicted global resistance will be equal to the squash load or yield moment.

Having the pure critical forces/moments determined, the resistances can be predicted separately for the three characteristic mode classes, by using Eqs. (16)- 20, and 21- 25 . The results of the calculations are summarized in Table 6 As it can be seen from the table, the proposed design approach based on FEM buckling modes identified by cFSM base functions leads to practically the same design resistances as the more classical approach based on regular FSM calculation. It is to highlight, however, that the proposed FEM-based approach is potentially more general, which is demonstrated by the partially restrained 2000-mm-long column problem, which cannot be handled (or can be handled with significant approximation) with the FSMbased approach. The FEM based design approach suggests that the applied light springs at the middle of the column yields to a non-negligible resistance increase, due to the increased resistance to global (flexural) buckling.

\section{Conclusions}

In this paper a novel design approach is presented and illustrated by some numerical examples which demonstrate both its advantages and difficulties.

The proposed design approach includes the following major steps: (i) buckling analysis carried out on a realistic model of the thin-walled member, by using shell finite element analysis, (ii) determination of the cFSM base functions, (iii) identification of the calculated multiple buckling modes which leads to the lowest critical forces/moments separately for the characteristic buckling modes, (iv) resistance prediction, which is proposed to do by the direct strength method.

Compared to other design approaches the proposed design approach has some definite advantages. Since the linear elastic buckling analysis is performed on a shell finite element model, a realistic model of the structural member is possible, which, potentially, can handle any irregularities (e.g., special support conditions, certain cross-section changes, holes, various loading, etc.) which cannot be handled by FSM. At the same time, the mode identification is fully automatic, does not involve any subjective decision from the engineer. It can be interesting to mention that the proposed FEM-based design approach has potential advantages even for regular cases (that could be handled by FSM), since FEM buckling analysis typically leads to several buckling modes where modes with different wave-lengths are combined (which cannot happen in the semi-analytical FSM). The ability of handling these combined modes is a definite advantage, even though it requires further research.

Comparisons on basic examples, as also presented in this paper, justify the applicability of the method. $\mathrm{C}$ and $\mathrm{Z}$ section beams and columns with various support conditions have been tested with reasonable results. Nevertheless, further development is necessary due to the difficulties and uncertainties as follows.

- The system of linear equations is relatively large. Potentially, it could be reduced by applying smaller number of cFSM base functions, by eliminating e.g., base functions of $\mathrm{O}$ mode class, or some of the L mode class. Most likely a significant reduction of the problem size is possible, but it requires further studies.

- At the moment the cFSM base functions are sine functions for the transverse, cosine functions for the longitudinal displacements, see Eq. (1), which limits the end support conditions to hinged or "more rigid" supports. Other shape functions would be necessary so that arbitrary boundary conditions could be handled, which is an important area for further research.

- The proposed design method has the potential to handle members with holes. One of the practical difficulties is that so far only regular FSM and FEM meshes are used with identical cross-section discretization. In case of members with holes a more general FEM mesh is necessary, thus, independent FEM 
Tab. 5. Nominal pure critical forces/moments for the selected models

\begin{tabular}{|c|c|c|c|c|c|c|c|c|}
\hline $\begin{array}{l}\text { Length } \\
\text { [mm] }\end{array}$ & Model & $\begin{array}{l}\text { FSM/ } \\
\text { FEM }\end{array}$ & Mode \# & $\begin{array}{c}P_{\text {cre }}[\mathrm{kN}] \\
M_{\text {cre }}[\mathrm{kNm}]\end{array}$ & Mode \# & $\begin{array}{c}P_{\mathrm{crl}}[\mathrm{kN}] \\
M_{\mathrm{crl}}[\mathrm{kNm}]\end{array}$ & Mode \# & $\begin{array}{c}P_{\text {crd }}[\mathrm{kN}] \\
M_{\text {crd }}[\mathrm{kNm}]\end{array}$ \\
\hline \multirow{4}{*}{800} & \multirow{2}{*}{ Column } & FSM & & - & & 80.64 & & 153.48 \\
\hline & & FEM & - & - & 1 & 78.13 & 11 & 162.76 \\
\hline & \multirow{2}{*}{ Beam } & FSM & & - & & 26.51 & & 20.50 \\
\hline & & FEM & - & - & 2 & 23.19 & 1 & 20.77 \\
\hline \multirow{5}{*}{2000} & \multirow{2}{*}{ Column } & FSM & & 158.29 & & 80.64 & & 153.48 \\
\hline & & FEM & 27 & 157.36 & 1 & 78.05 & 26 & 154.27 \\
\hline & Restrained column & FEM & 40 & 245.20 & 1 & 77.59 & 26 & 154.83 \\
\hline & \multirow{2}{*}{ Beam } & FSM & & 23.41 & & 26.51 & & 20.50 \\
\hline & & FEM & 2 & 22.15 & 6 & 23.46 & 1 & 19.36 \\
\hline \multirow{4}{*}{3600} & \multirow{2}{*}{ Column } & FSM & & 51.85 & & 80.64 & & 153.48 \\
\hline & & FEM & 1 & 51.57 & 2 & 78.08 & 50 & 156.34 \\
\hline & \multirow{2}{*}{ Beam } & FSM & & 7.52 & & 26.51 & & 20.50 \\
\hline & & FEM & 1 & 6.65 & 2 & 22.01 & 3 & 21.77 \\
\hline
\end{tabular}

Tab. 6. Predicted resistances for the selected models

\begin{tabular}{|c|c|c|c|c|c|c|}
\hline $\begin{array}{l}\text { Length } \\
{[\mathrm{mm}]}\end{array}$ & Model & $\begin{array}{l}\text { FSM/ } \\
\text { FEM }\end{array}$ & $\begin{array}{c}P_{\text {ne }}[\mathrm{kN}] \\
M_{\text {ne }}[\mathrm{kNm}]\end{array}$ & $\begin{array}{c}P_{\mathrm{nl}}[\mathrm{kN}] \\
M_{\mathrm{nl}}[\mathrm{kNm}]\end{array}$ & $\begin{array}{c}P_{\mathrm{nd}}[\mathrm{kN}] \\
M_{\mathrm{nd}}[\mathrm{kNm}]\end{array}$ & $\begin{array}{c}P_{\mathrm{n}}[\mathrm{kN}] \\
M_{\mathrm{n}}[\mathrm{kNm}]\end{array}$ \\
\hline \multirow{4}{*}{800} & \multirow{2}{*}{ Column } & FSM & 265.26 & 149.40 & 156.63 & 149.40 \\
\hline & & FEM & 265.26 & 147.72 & 160.97 & 147.72 \\
\hline & \multirow{2}{*}{ Beam } & FSM & 14.11 & 14.11 & 12.49 & 12.49 \\
\hline & & FEM & 14.11 & 14.06 & 12.55 & 12.55 \\
\hline \multirow{5}{*}{2000} & \multirow{2}{*}{ Column } & FSM & 131.54 & 94.82 & 156.63 & 94.82 \\
\hline & & FEM & 131.00 & 93.51 & 157.01 & 93.51 \\
\hline & Restrained column & FEM & 168.67 & 110.04 & 157.28 & 110.04 \\
\hline & \multirow{2}{*}{ Beam } & FSM & 13.05 & 13.05 & 12.49 & 12.49 \\
\hline & & FEM & 12.90 & 12.90 & 12.27 & 12.27 \\
\hline \multirow{4}{*}{3600} & \multirow{2}{*}{ Column } & FSM & 45.47 & 45.47 & 156.63 & 45.47 \\
\hline & & FEM & 45.23 & 45.23 & 157.99 & 45.23 \\
\hline & \multirow{2}{*}{ Beam } & FSM & 7.52 & 7.52 & 12.49 & 7.52 \\
\hline & & FEM & 6.65 & 6.65 & 12.73 & 6.65 \\
\hline
\end{tabular}


and FSM meshes are unavoidable. This generalization does not mean any theoretical challenge, but requires further development work.

- The practical application of the method requires various matrix manipulations, which ultimately requires special software. At the moment such software is not publicly available, but a user friendly computer program is under development by the Authors.

\section{Acknowledgments}

The presented work has been performed under the partial financial support of the OTKA K62970 project and the OTKA T049305 project of the Hungarian Scientific Research Fund.

\section{References}

1 Fóti P, Dunai L, Test based design method of moment resisting joints in cold-formed structures, International Colloquium on Stability and Ductility of Steel Structures, 2002, pp. 211-218. Prof. O. Halász Memorial Session.

2 Dudás A, Light steel structures in residential house construction, Per. Pol. Civil Eng. 47 (2003), no. 1, 133-136.

3 Jakab G, Dunai L, Interaction phenomena of cold-formed truss members and joints, Proceedings of the Fifth International Conference on Coupled Instabilities in Metal Structures (CIMS 2008), 2008, pp. 515-522.

4 Erdélyi Sz, Dunai L, Behaviour of a new type of composite connection, Per. Pol. Civil Eng. 48 (2004), no. 1-2, 89-100.

5 Cheung YK, Finite Strip Method in Structural Analysis, Pergamon Press, 1976.

6 Papangelis JP, Hancock GJ, Computer analysis of thin-walled structural members, Computers \& Structures 56 (1995), no. 1, 157-176.

7 Schafer BW, Cold-formed steel behavior and design: analytical and numerical modeling of elements and members with longitudinal stiffeners, Cornell University, Ithaca, NY, USA, 1997. PhD thesis.

8 CUFSM Elastic Buckling Analysis of Thin-Walled Members by Finite Strip Analysis v3.12, http://www.ce.jhu.edu/bschafer, 2006. Free finite strip software for elastic buckling.

9 Ádány S, Schafer BW, Buckling mode decomposition of singlebranched open cross-section members via Finite Strip Method: derivation, Thin-Walled Structures 44 (2006), no. 4, 563-584, DOI 10.1016/j.tws.2006.03.013.

10 _ Buckling mode decomposition of single-branched open cross-section members via Finite Strip Method: application and examples, Thin-Walled Structures 44 (2006), no. 4, 585-600, DOI 10.1016/j.tws.2006.03.014.

11 _ A full modal decomposition of thin-walled, single-branched open cross-section members via the constrained finite strip method, Journal of Constructional Steel Research 64 (2008), no. 1, 12-29, DOI 10.1016/j.jcsr.2007.04.004.

12 Ádány S, Buckling mode classification of members with open thin-walled cross-sections by using the Finite Strip Method, Johns Hopkins University, 2004. http://www.ce.jhu.edu/bschafer.

13 Schafer BW, Ádány S, Buckling analysis of cold-formed steel members using CUFSM: conventional and constrained finite strip methods, Orlando, FL, USA, 2006. Eighteenth International Specialty Conference on Cold-Formed Steel Structures.

14 Casafont M, Marimon F, Pastor MM, Calculation of pure distortional elastic buckling loads of members subjected to compression via the finite element method, Thin-Walled Structures (2009). In Press.

15 Ádány S, Joó AL, Schafer BW, Approximate Identification of the buckling modes of thin-walled columns by using the CFSM modal base functions, Lis- bon, Portugal, 2008. International Colloquium on Stability and Ductility of Steel Structures.

16 _ Identification of FEM buckling modes of thin-walled columns by using cFSM base functions, Sydney, Australia, 2008. Fifth International Conference on Coupled Instabilities in Metal Structures.

17 Silvestre N, Camotim D, First-order generalised beam theory for arbitrary orthotropic materials, Thin-Walled Structures 40 (2002), no. 9, 755-789, DOI 10.1016/S0263-8231(02)00025-3.

18 , Second-order generalised beam theory for arbitrary orthotropic materials, Thin-Walled Structures 40 (2002), no. 9, 791-820, DOI 10.1016/S0263-8231(02)00026-5.

19 EN 1993-1-3:2006, Eurocode 3, Design of Steel Structures, Part 1-3, 2006. General rules, Supplementary rules for cold-formed thin gauge members and sheeting.

20 Specification for the Design of Cold-Formed Steel Structural Members, American Iron and Steel Institute (AISI), Washington, DC, USA, 1996.

21 Schafer BW, Review: The Direct Strength Method of Cold-formed Steel Member Design, Proceedings of International Colloquium on Stability and Ductility of Steel Structures, 2006, pp. 49-66.

22 North American Specification for the Design of Cold-Formed Steel Structures, American Iron and Steel Institute, Washington, DC, USA, 2001.

232004 Supplement to the North American Specification for the Design of ColdFormed Steel Structures, American Iron and Steel Institute, Washington, DC, USA, 2004.

24 Jakab G, Joó AL, Dunai L., Experimental studies of C/Z profiles compression members, Proceedings 4th International of Coupled Instabilities in Metal Structures, 2004, pp. 429-438.

25 ANSYS Release 11.0 Documentation, Ansys Inc., 2007.

26 MatLab Version 7.1.0246 Documentation, The Mathwork Inc., 2005. 